\title{
Protocolo Baseado em Métricas de Redes Complexas para Mitigação de Tempestade de Broadcast
}

\author{
Efrem E. de Oliveira Lousada ${ }^{1,3}$, Carlos R. Storck ${ }^{2,3}$, \\ Raquel A. F. Mini $^{3}$, Fátima Duarte-Figueiredo ${ }^{3}$ \\ ${ }^{1}$ Instituto Federal de Minas Gerais (IFMG) \\ ${ }^{2}$ Centro Federal de Educação Tecnológica de Minas Gerais (CEFET-MG) \\ ${ }^{3}$ Pontifícia Universidade Católica de Minas Gerais (PUC Minas) \\ efrem.lousada@ifmg.edu.br, storck@cefetmg.br, \\ \{raquelmini, fatimafig\}@pucminas.br
}

\begin{abstract}
The broadcast storm is characterized by unwanted packet retransmission, causing frequent flooding, contention, and collisions that compromise network performance. This paper presents a new protocol for the dissemination of messages in vehicular networks through the observation and analysis based on complex network metrics. It is called CN-vP (Complex Network - vehicular Protocol). In order to mitigate the sending of unnecessary messages, the present work combined probabilistic and delay approaches together with a better knowledge of the network, in particular, the neighboring vehicles of a certain transmitter. Once the three best relays have been chosen, by means of the probabilistic calculation, waiting intervals are estimated, for each retransmission, in order to avoid packet flooding. The analysis of the results shows that the solution developed allows a more assertive decision making for the dissemination of messages in vehicle networks, thus mitigating the problem of broadcast storm.
\end{abstract}

Resumo. A tempestade de broadcast é caracterizada pela retransmissão indesejada de pacotes, ocasionando inundações, contenções e colisões frequentes que comprometem o desempenho da rede. Este artigo apresenta um protocolo para a disseminação de mensagens em redes veiculares através da observação e da análise baseada em métricas de redes complexas, intitulado $C N-v P$ (Complex Network - vehicular Protocol). Com o objetivo de mitigar o envio de mensagens desnecessárias, o presente trabalho combinou abordagens probabilísticas juntamente com maior conhecimento da rede, em particular, os veículos vizinhos de um determinado transmissor. Uma vez escolhidos os três melhores retransmissores, através do cálculo probabilístico, sao estimados intervalos de espera, para cada retransmissão, de maneira a evitar inundações de pacotes. A análise dos resultados mostra que a solução desenvolvida permite uma tomada de decisão mais assertiva para a disseminação de mensagens em redes veiculares, mitigando, assim, o problema de tempestade de broadcast.

\section{Introdução}

VANETs são um caso especial de rede ad hoc na qual os veículos, equipados com processamento e dispositivos de comunicação sem fio, podem criar uma rede enquanto 
se movem ao longo de suas trajetórias. Vários serviços se tornam possíveis com a implantação das VANETs. Um dos principais envolve a segurança durante a condução dos veículos [Hartenstein and Laberteaux 2008]. Detectar condições críticas das estradas, como acidentes, condições adversas da via e frenagens bruscas, e informar de forma rápida para o máximo possível de veículos é o principal objetivo deste tipo de serviço. Dessa forma, é necessária uma cooperação entre veículos para retransmitir a mensagem em uma ampla área, de maneira que uma disseminação efetiva de tal mensagem é dependente de retransmissões de múltiplos saltos [Amadeo et al. 2016]. A tempestade de broadcast ocorre quando existem tantas mensagens de difusão na rede que elas se aproximam ou superam a capacidade da largura de banda disponível. Durante uma tempestade de broadcast, o nível de contenção e colisão de pacotes aumenta significamente, devido a um número excessivo de encaminhamentos [Hafi et al. 2017].

Evitar ou mitigar o problema de tempestade de broadcast, em VANETs, tem sido um grande desafio para pesquisadores. Algoritmos probabilísticos e baseados em espera são propostos e usualmente empregados. Algoritmos probabilísticos realizam a decisão sobre encaminhamento com base em um valor de limiar. Para adaptar a dinâmica da rede, este parâmetro deve ser gerado dinamicamente e definido de acordo com os melhores valores de probabilidade de encaminhamento dos vizinhos, denominado como $p$. Dessa forma, busca-se garantir apenas as transmissões necessárias e evitar retransmissões redundantes. Esse limiar é gerado com base em entradas antigas e atuais da probabilidade $p$, fazendo-se uso da estatística Média Móvel Exponencial Ponderada (Exponentially Weighted Moving Average - EWMA). Algoritmos baseados em espera criam momentos diferentes para transmissão na rede. No protocolo E-Probt [Lima and Júnior 2016], após o cálculo de $p$, para os três melhores retransmissores, estima-se os intervalos de espera, para encaminhamento, para cada um. Este procedimento evita encaminhamentos redundantes, pois após o tempo de espera, o nó verifica se a informação foi retransmitida por outro nó.

Neste contexto, este trabalho apresenta um protocolo para a tomada de decisão sobre o encaminhamento de pacotes que mitigue o problema de tempestade de broadcast. Um protocolo multihop, combinando características de protocolos probabilísticos e de intervalos de espera é apresentado, visando a melhoria na eficiência do uso de recursos de rede e evitando a disseminação de mensagens desnecessárias entre veículos. No protocolo proposto por este trabalho, serão analisadas as diferentes métricas dos vizinhos: grau do vértice, sobreposição topológica e persistência das arestas, baseadas em métricas de redes complexas [Rezende et al. 2011], para a tomada de decisão sobre encaminhamento de pacotes. Essas métricas são modeladas matematicamente através de equações probabilísticas. Os resultados obtidos pelas equações são classificados e combinados. O resultado da combinação indicará a decisão sobre encaminhamento. Resultados de simulações mostraram um ganho na taxa de entrega em cenários de trânsito leve (20 veículos $/ \mathrm{km}$ ) e moderado (30 veículos $/ \mathrm{km}$ ) e um comportamento semelhante ao protocolo E-Probt [Lima and Júnior 2016] nos demais cenários. Além disso, o protocolo proposto apresenta uma diminuição na taxa de redundância em comparação aos protocolos existentes na literatura com equivalentes taxas de entrega. $\mathrm{O} \mathrm{CN}-\mathrm{vP}$ diminui consideravelmente a quantidade de transmissões necessárias em relação ao E-Probt.

Este trabalho está organizado da seguinte forma: a Seção 2 apresenta os trabalhos 
relacionados; a Seção 3 descreve o protocolo proposto; a Seção 4 apresenta a metodologia adotada; a Seção 5 avalia os resultados experimentais da pesquisa; e a Seção 6 discorre as conclusões e proposições a trabalhos futuros.

\section{Trabalhos Relacionados}

No trabalho desenvolvido em [Lima et al. 2015], os autores apresentam um protocolo probabilístico e temporal para a mitigação do problema de tempestade de broadcast em VANETs. O protocolo utiliza como base a quantidade de vizinhos em comum, bem como a distância entre emissor e receptor. Em [Lima and Júnior 2016], são usadas recompensas e penalidades para veículos vizinhos, aplicando-se a Teoria dos Jogos. Ambos os trabalhos, [Lima et al. 2015] e [Lima and Júnior 2016], apresentaram em seus resultados altas taxas de redundância, próximas as do protocolo Blind Flooding, sendo as maiores entre os trabalhos comparados. Trabalhos anteriores para mitigar o problema de tempestade de broadcast foram relacionados em [Lima and Júnior 2016], como mostra a Tabela 1.

Tabela 1. Protocolos de Broadcast

\begin{tabular}{ll}
\hline Protocolo & Problemas \\
\hline AutoCast & Não funciona quando o valor de Nh é menor que 5 \\
Blind Flooding & Não escalável e produz a tempestade de broadcast \\
Irresponsible Forwarding & Descarta irresponsavelmente os pacotes \\
Weighted p-persistence & Considera um raio de transmissão fornecido por veículos e não verifica pacotes \\
& encaminhados por outros veículos na rede \\
E-probT & Taxa de entrega inferior a $75 \%$ em cenários de tráfego moderado e baixo \\
\hline
\end{tabular}

O AutoCast adota uma estratégia semelhante ao protocolo OAPB [Alshaer and Horlait 2005], que faz uso de zonas vizinhas para o cálculo da probabilidade $p$. O valor calculado para $p$ depende da quantidade de veículos vizinhos alcançáveis por uma ou duas transmissões. Após o cálculo de $p$, o protocolo calcula intervalos de tempo de espera para repropagar o pacote. Este valor é um resultado da multiplicação de duas variáveis, $p$ e uma variável aleatória, e uma constante, máximo intervalo de espera. O AutoCast calcula a probabilidade de encaminhamento com diferentes densidades de vizinhos. A probabilidade é baseada no número de veículos da vizinhança alcançáveis através de 1 (um) salto (Nh). Em [Panichpapiboon and Pattara-atikom 2012], é feita uma crítica à esse protocolo, pelo fato de não funcionar quando o valor de $N h$ for menor que 5 . Além disso, através dos resultados obtidos em experimentos, esse protocolo apresentou uma baixa taxa de entrega, não atingindo $25 \%$.

A técnica BF (Flood Blind) é considerada a maneira mais simples de enviar dados por difusão. Ao receber um pacote, o nó verifica se o mesmo já foi recebido, se sim, descarta o pacote; caso contrário, retransmite o mesmo sem qualquer critério de seleção. Pesquisas mostram que esta técnica é ineficiente, não possui escalabilidade e produz a indesejada tempestade de broadcast [Saeed et al. 2019].

A primeira estratégia do IF (Irresponsible Forwarding) leva em consideração a distância entre emissor e receptor. A segunda estratégia é baseada na distribuição estatística dos veículos para o cálculo de $p$. Ao receber um pacote, o veículo receptor avalia a probabilidade de existir um outro veículo mais distante do emissor com uma melhor probabilidade de encaminhamento. Se não existir, este veículo faz o encaminhamento. Caso contrário, o veículo descarta irresponsavelmente o pacote e não 
o encaminha. Com base nos resultados obtidos, este protocolo possui a menor taxa de entrega entre os trabalhos existentes, próximo a $10 \%$.

O Weighted p-persistence utiliza a distância do possível receptor e sua faixa de transmissão para calcular a probabilidade de encaminhamento. Nos resultados apresentados, quanto mais longe do emissor, maior a probabilidade de encaminhamento [Sharshembiev et al. 2018]. Todos os trabalhos apresentados nesta Seção possuem uma taxa de entrega inferior a $75 \%$ em cenários de tráfego moderado e baixo. Além disso, para alcançar uma taxa de entrega próxima aos $90 \%$ nos demais cenários, os protocolos apresentados necessitam de um aumento significativo no número de retransmissões. Este aumento impacta diretamente a taxa de redundância, uma vez que também implica em aumentar a duplicação de pacotes. Dessa forma, a principal motivação para o trabalho proposto é alcançar uma boa taxa de entrega e uma baixa taxa de redundância, reduzindo o número de transmissões necessárias para atingir o maior número de veículos.

\section{Abordagem Proposta}

$\mathrm{O}$ protocolo $\mathrm{CN}-\mathrm{vP}$ visa minimizar o número de veículos retransmissores e a redundância de pacotes. Manter as relações entre taxa de entrega, número de retransmissões e redundância de pacotes estáveis, com variação da densidade de veículos na rede, é o maior desafio das soluções anteriores. O funcionamento do $\mathrm{CN}$-vP é mostrado no Algoritmo 1.

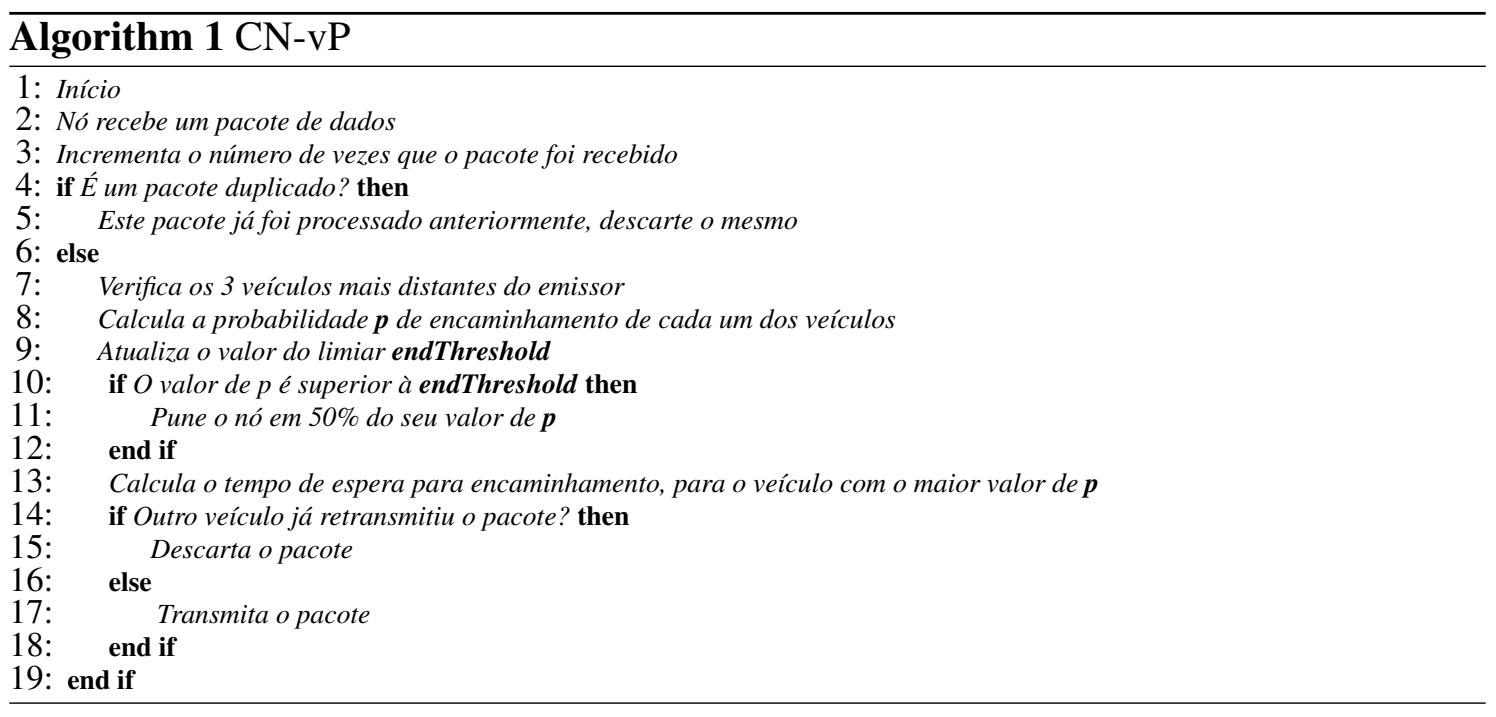

Os veículos que devem receber os pacotes são os mais distantes do emissor e os mais bem classificados levando em consideração a eficiência. A posição do veículo na rede é fator prepoderante para ser escolhido ou não para receber o pacote. Basedo na proposta de [Lima and Júnior 2016], são escolhidos os três veículos mais distantes do emissor para ranqueamento.

A definição do quão um veículo é eficiente para realizar a retransmissão é dada por uma função de probabilidade que leva em consideração o grau do vértice, a sobreposição topológica e a persistência das arestas. A probabilidade de retransmissão $p$ calculada, que é definida pela equação 1 na próxima subseção, é comparada com um valor de um limiar gerado de acordo com uma média móvel exponencialmente ponderada. Caso o valor de $p$, de um dos três veículos selecionados, seja maior que o limiar, esse veículo é penalizado 
e pode ir para último lugar no ranking. O melhor ranqueado recebe o pacote para retransmitir. A escolha dos componentes para calcular a probabilidade de retransmissão $p$, baseou-se na importância de um determinado veículo para a retransmissão de pacotes na rede. As seguintes métricas abaixo, de redes complexas, são utilizadas pelo protocolo $\mathrm{CN}-\mathrm{vP}$ da seguinte forma:

- Grau do vértice: vértices que apresentam um grau elevado são os mais ativos na rede, e podem atuar como um canal para a troca de informações;

- Sobreposição topológica: retrata a tendência de existir vértices com vizinhos partilhados. Sendo assim, quanto menor a quantidade de vizinhos compartilhados, maior a possibilidade de se atingir um número maior de veículos;

- Persitência das arestas: define o número de vezes que dois vértices se encontram na mesma janela temporal. Veículos que estão em uma mesma janela temporal possuem maior probabilidade de sucesso ao trocar informações.

$\mathrm{O}$ valor de $p$ é calculado pelo produto da probabilidade associada ao Grau do Vértice $(p G V)$, da probabilidade associada a Sobreposição Topológica ( $p S T)$ e da probabilidade associada a Persitência das Arestas $(p P A)$, formulado pela equação 1:

$$
p=p G V \times p S T \times p P A
$$

Para o cálculo de $p G V$ de um determinado veículo, é necessário que o veículo saiba o grau do vértice de todos os seus vizinhos, este valor é mantido e atualizado na sua tabela de vizinhos. O veículo, então, realiza a razão entre o seu grau de vértice $(g V)$ e o somatório dos graus do vértice de seus vizinhos $(g V V)$, conforme ilustrada na equação 2 .

$$
p G V=\frac{g V}{g V V}
$$

Durante a disseminação de dados é interessante a seleção de veículos que possuam o mínimo possível de vizinhos em comum. O cálculo de $p S T$ é dado por 1 (um) menos a divisão do número de vizinhos em comum $(n V C)$ pela soma do número de vizinhos do transmissor $(n V T)$ com o número de vizinhos do receptor $(n V R)$. A equação 3 exibe a formulação descrita.

$$
p S T=1-\frac{n V C}{n V T+n V R}
$$

$O$ veículo transmissor considera os dois segundos anteriores a escolha do receptor para calcular o valor de $p P A$. Se a aresta manteve-se pelos dois segundos a $p P A$ recebe o valor 1 , se apareceu somente no último segundo recebe o valor 0,5 e caso no último segundo não tenha aresta, receberá o valor 0 , evitando assim que o veículo seja escolhido como receptor. Após o cálculo de $p$ para os três veículos mais distantes, é necessário verificar se os valores são superiores ao valor de limiar. Caso o valor seja maior, o mesmo é penalizado em $50 \%$, alterando para a última posição o veículo penalizado.

Os cálculos do limiar e do intervalo de espera, baseiam-se na metodologia adotada pelo protocolo E-Probt apresentado em [Lima and Júnior 2016]. O valor inicial de limiar, chamado de avgThreshold, é calculado com a média de $p$ para os três veículos mais distantes do transmissor. Com o objetivo de refinar este valor, o mesmo passa por um processo de média móvel exponencial ponderada, que considera valores anteriores e 
atuais para cálculo dos novos limiares. Com isso tenta-se eliminar possíveis disparidades, bem como possíveis erros na estimativa realizada. O valor do intervalo de espera $(w T)$ é importante para criar momentos diferentes para transmissão de dados na rede. Este procedimento evita encaminhamentos redundantes, pois após o tempo de espera o veículo verifica se a informação foi retransmitida por outro nó. Neste caso, o pacote é descartado.

\section{Metodologia}

O protocolo $\mathrm{CN}$-vP foi testado através do simulador de eventos discretos em rede ns3 , que foi projetado para simular diferentes tecnologias e cenários. $\mathrm{O}$ modelo de tráfego adotado no trabalho foi criado através do Simulation of Urban Mobility (SUMO), consistindo em um rodovia com 2 pistas e veículos se movendo na mesma direção, da esquerda para a direita. Para que veículos cruzem com outros veículos durante os seus trajetos, velocidades distintas foram adotadas para tornar possível as ultrapassagens. $\mathrm{O}$ cenário simulado é apresentado na Figura 1.

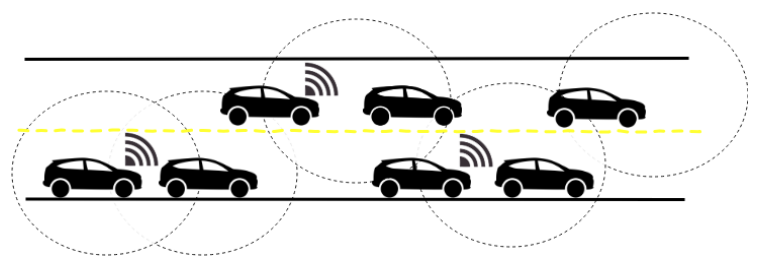

Figura 1. Cenário simulado.

A Tabela 2 apresenta os parâmetros usados nas simulações. As densidades de veículos foram definidas e classificadas como trânsito leve (20 veículos/km), trânsito moderado (30 veículos $/ \mathrm{km}$ ), trânsito pesado (60 veículos $/ \mathrm{km}$ ) e trânsito congestionado (90 e 120 veículos $/ \mathrm{km}$ ) [Wisitpongphan et al. 2007]. Cada simulação foi executada com a adoção de 300 segundos como tempo total. Inicialmente, um intervalo de aquecimento da rede, termo conhecido como warm-up, é realizado por 10 segundos. Esse período é usado para uma melhor caracterização do valor de limiar e cálculo de intervalos de espera utilizados na simulação, antes do início da medição das métricas.

Tabela 2. Parâmetros de simulação

\begin{tabular}{lll}
\hline Parâmetro & Valor & Descrição \\
\hline vehicles & 300 & Número de Veículos \\
density & $20,30,60,90$ e 120 & Veículos/km \\
speed & $36,54,72$ e $90 \mathrm{~km} / \mathrm{h}$ & Velocidades distintas dos veículos \\
dataPacket & 48 bytes & Tamanho do pacote \\
sendingRate & 1 pacote/segundo & Taxa de envio de pacotes \\
propagation & Nakagami & Modelo de propagação \\
transport & UDP & Protocolo de transporte \\
MAC/PHY & $802.11 \mathrm{p}$ & Sistema de comunicação veicular \\
transmision & $1 \mathrm{~km}$ & Faixa de transmissão \\
simTime & 300 segundos & Tempo total da simulação \\
interval & $95 \%$ & Intervalo de confiança \\
\hline
\end{tabular}

As métricas escolhidas para comparar os protocolos, descritas em [Panichpapiboon and Pattara-atikom 2012, Paula et al. 2014, Lima et al. 2015], foram a taxa de entrega, o número de transmissões e a taxa de redundância. A taxa de entrega de pacotes normalizada pertence ao intervalo $[0,1]$ em $\mathbb{R}$. Ela é a relação entre 
a quantidade de veículos receptores dos pacotes transmitidos e o número de veículos da rede. Busca-se entregar um pacote a todos os nós da rede. Assim, apresentam um melhor desempenho os protocolos com as maiores taxas de entrega. O número de transmissões é uma média da quantidade máxima de transmissões que cada pacote realiza. Busca-se entregar o pacote ao maior número de veículos na rede com o menor número de transmissões possíveis, uma vez que o consumo de energia e de largura de banda se torna menor. Por último, a taxa de redundância indica a quantidade média de duplicações de cada pacote que é recebida pelos veículos. Apresentam um melhor desempenho, os protocolos com menores taxas de duplicações. Os resultados da análise obtida nos experimentos são apresentados na seção a seguir.

\section{Resultados}

\subsection{Taxa de entrega}

A Figura 2 apresenta, através do eixo y, a taxa de entrega obtida pelo protocolo $\mathrm{CN}$ vP frente aos outros protocolos com diferentes densidades de veículos, apresentadas pelo o eixo x. Pelas simulações, o protocolo $\mathrm{CN}$-vP apresentou um comportamento constante, próximo a $90 \%$ de veículos que receberam o pacote, em todas as densidades de veículos, mesmo quando o trânsito está leve, dificultando a propagação dos pacotes. Os demais protocolos possuem limitações em tráfego com poucos veículos. Considerando o intervalo de confiança de $95 \%$, em cenários de baixa densidade de veículos (trânsito leve), o CN-vP foi superior aos outros protocolos, apresentando ainda um ganho de $18 \%$ quando comparado ao protocolo E-Probt [Lima and Júnior 2016]. Em cenários de trânsitos pesados ou congestionados, o $\mathrm{CN}-\mathrm{vP}$ obteve um comportamento equivalente ao protocolo E-Probt. O ganho apresentado pelo $\mathrm{CN}$-vP é devido ao encaminhamento de pacotes associado ao uso de métricas de redes complexas, capazes de conhecerem os seus melhores vizinhos juntamente com as respectivas probabilidades de retransmissão.

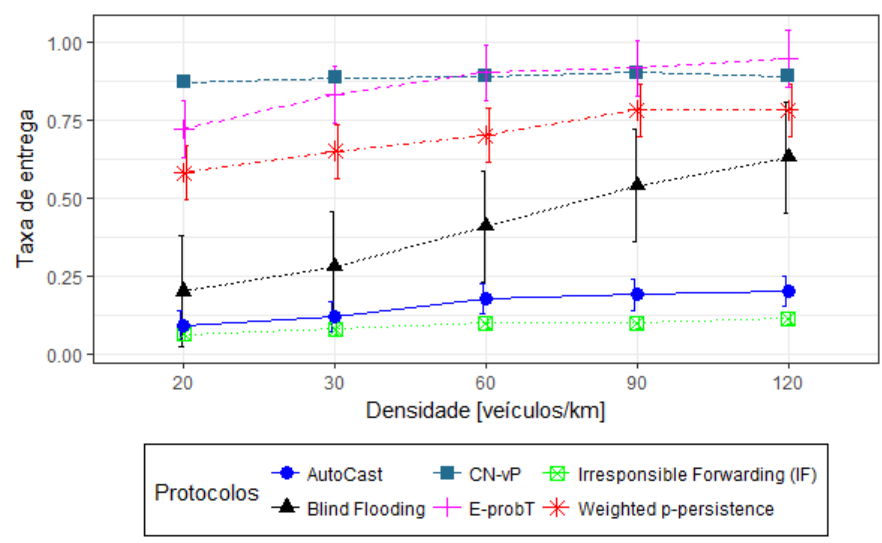

Figura 2. Taxa de entrega

Entre os protocolos analisados, as piores taxas de entrega são apresentadas pelos protocolos Blind Flooding, AutoCast e Irresponsible Forwarding. Este resultado é justificado pelo fato de que os mesmos não possuem quaisquer mecanismos sobre a verificação de encaminhamento por parte de outros veículos. A assertividade dos protocolos é dependente de uma modelagem quase perfeita da tendência correta de encaminhamento. O protocolo Weighted p-persistence apresentou uma taxa de entrega 
regular. O mesmo adota como técnica a verificação da distância para o possível emissor, sendo a sua principal variável durante o cálculo da probabilidade de retransmissão. Mesmo sendo uma abordagem mediana, diversos veículos da rede ainda ficam sem receber o pacote. A razão do não recebimento dos pacotes é explicado, primeiramente, pelo fato de que o protocolo Weighted p-persistence considera um raio de transmissão preenchido por veículos, o que nem sempre se torna verdade. A segunda razão é a não verificação do encaminhamento de pacotes por outros veículos pelo protocolo.

\subsection{Número de transmissões}

Conseguir atingir o maior número de veículos na rede com o menor número de transmissões é o grande desafio das soluções propostas. Número de transmissões deve ser analisado juntamente com a taxa de entrega, uma vez que mantendo uma boa relação entre as duas, pode-se atingir um número alto de veículos sem comprometer a capacidade da largura de banda disponível. O número de transmissões é apresentado na Figura 3.

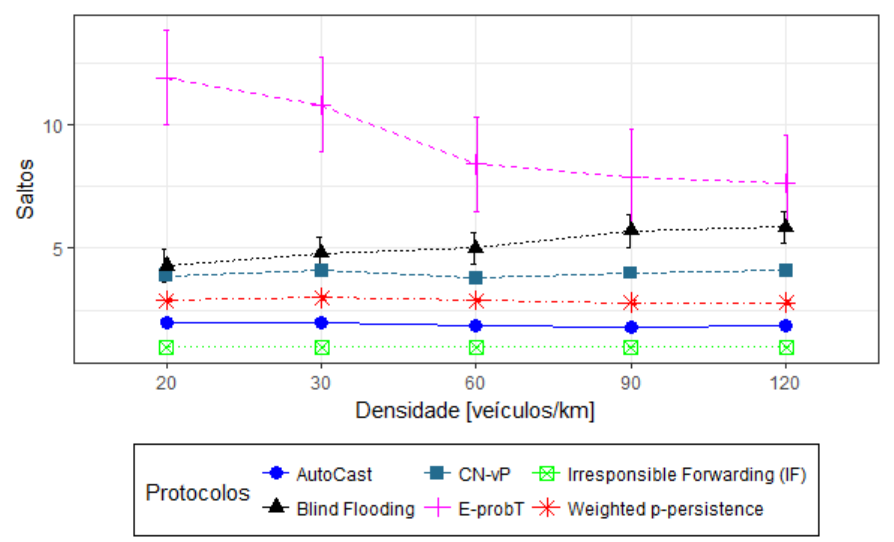

Figura 3. Número de transmissões

Os protocolos Weighted p-persistence, AutoCast e Irresponsible Forwarding apresentaram valores consideravelmente baixos de transmissões. Estes protocolos não verificam o encaminhamento de pacotes realizados por outros nós, o que deixa os veículos mais distantes do emissor sem receber muitos pacotes. Por não receberem os pacotes, estes não são contabilizados como transmissões realizadas. O Blind Flooding obtém uma quantidade de transmissões mediana. Muitos veículos distantes do emissor também não recebem os pacotes devido a alta colisão que ocorre no processo de encaminhamento. $\mathrm{O}$ problema destes protocolos ficam evidentes quando se analisa a relação entre veículos que receberam os pacotes e o número de transmissões realizadas durante a execução. Eles realizam poucas retransmissões, mas também possuem uma taxa de entrega baixa. Os protocolos com as melhores taxas de entrega possuem comportamentos diferentes quando se analisa o número de transmissões. O E-Probt necessita de mais retransmissões para atingir a taxa de entrega próxima a 90\%. Enquando o $\mathrm{CN}-\mathrm{vP}$ necessita de aproximadamente $50 \%$ das transmissões do E-Probt para se obter taxas similares e até melhores quando o trânsito está leve ou moderado.

\subsection{Taxa de redundância}

A taxa de redundância, definida pela equação 10, mostra o quão eficiente o protocolo é para escolher corretamente os nós retransmissores de pacotes, evitando que um mesmo 
veículo receba mais de uma vez o mesmo pacote. Assim como a métrica número de transmissões, esta métrica deve ser avaliada em conjunto à taxa de entrega. Não adianta obter uma taxa de redundância baixa se a taxa de entrega também for baixa. A taxa de redundância obtida pelo $\mathrm{CN}$-vP é exibida na Figura 4.

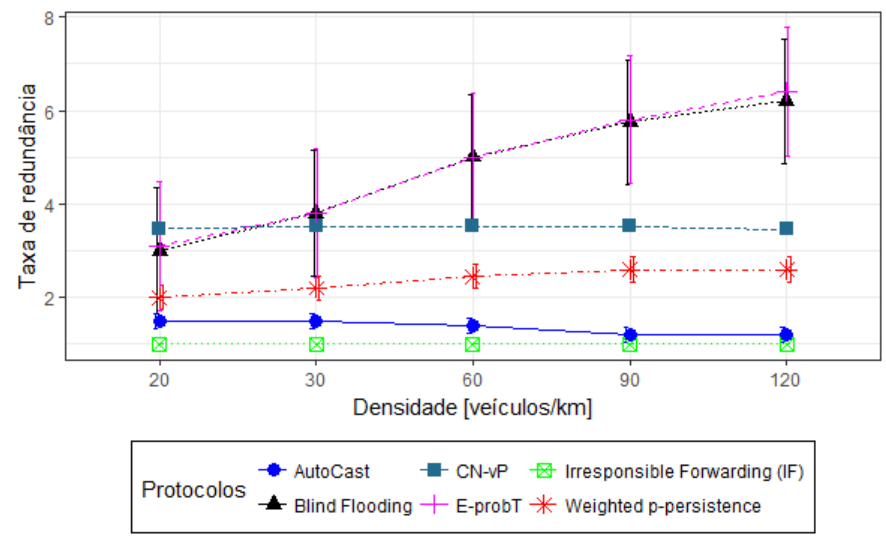

Figura 4. Taxa de redundância

Os protocolos AutoCast e Irresponsible Forwarding apresentaram taxas de redundância baixas, mas também as piores taxas de entrega. O protocolo Weighted ppersistence possui uma taxa mediana de redundância. Entretanto, uma taxa considerável de veículos continua sem receber os pacotes devido a sua média taxa de entrega. Blind Flooding e E-Probt possuem as piores taxas de redundância. Importante destacar que o protocolo Blind Flooding é o que mais produz pacotes. No entanto, as colisões existentes fazem com que muitos pacotes não sejam contabilizados pela métrica. O E-Probt, apesar de ter uma maior taxa de redundância entre os protocolos avaliados, possui uma taxa de entrega média de $90 \%$. A melhor relação entre a taxa de redundância e a taxa de entrega foi a do protocolo $\mathrm{CN}-\mathrm{vP}$ proposto, que apresentou uma redundância menor que os protocolos Blind Flooding e E-Probt. Ainda, o $\mathrm{CN}-\mathrm{vP}$ se assemelha com o comportamento dos resultados obtidos do Weighted p-persistence, mas com a melhor taxa de entrega, principalmente em ambientes de baixo tráfego ou moderado.

Por fim, um fator importante do protocolo $\mathrm{CN}$-vP é a sua estabilidade em todas as métricas avaliadas independente da densidade de veículos, justificada pelo melhor conhecimento da rede. Como pode ser observado nas três métricas avaliadas, a densidade não foi o fator preponderante para a dispersão dos resultados obtidos.

\section{Conclusões e Trabalhos Futuros}

A retransmissão de mensagens desnecessárias, ocasionada pela tempestade de broadcast, compromete o desempenho da rede como um todo, o que causa sérias consequências principalmente quando serviços críticos e de segurança são necessários. A proposta desenvolvida neste trabalho, intitulada de $\mathrm{CN}-\mathrm{vP}$, mitiga tal efeito através da análise da estrutura e do comportamento dinâmico da rede veicular, o que torna possível uma maior compreensão e decisões mais assertivas para encaminhamento de mensagens. Entre as técnicas empregadas pelo $\mathrm{CN}-\mathrm{vP}$, a escolha de veículos vizinhos retransmissores adotando métricas de redes complexas, combinada com um intervalo de espera, evitam encaminhamentos redundantes. 
Pelas simulações realizadas, o presente trabalho compara seus resultados com as soluções apresentadas na Seção 2. O protocolo $\mathrm{CN}-\mathrm{vP}$ melhorou a taxa de entrega em cenários de baixa densidade de veículos, além de manter uma taxa de entrega consideravelmente alta, diminuindo, assim, a taxa de redundância. Como trabalhos futuros são sugeridas a realização de testes com novos modelos de mobilidade, assim como a adoção de outras tecnologias de comunicação sem fio, por exemplo, redes celulares $5 \mathrm{G}$.

\section{Agradecimentos}

Os autores agradecem à Fapemig, à CAPES, ao IFMG, ao CEFET-MG e à PUC Minas pelo suporte e apoio financeiro recebidos.

\section{Referências}

Alshaer, H. and Horlait, E. (2005). An optimized adaptive broadcast scheme for intervehicle communication. In Vehicular Technology Conference, volume 5, page 2840-2844.

Amadeo, M., Campolo, C., and Molinaro, A. (2016). Information-centric networking for connected vehicles: a survey and future perspectives. IEEE Communications Magazine, 54(2):98-104.

Hafi, H., Abdou, W., and Merniz, S. (2017). Adaptive-segmentation and flexible-delay based broadcasting protocol for vanets. In Wired/Wireless Internet Communications (WWIC 2017), volume 10372, pages 207-218.

Hartenstein, H. and Laberteaux, L. (2008). A tutorial survey on vehicular ad hoc networks. IEEE Communications Magazine, 46(6):164-171.

Lima, D. S. and Júnior, J. C. (2016). E-probt: A new approach to mitigate the broadcast storm problem in vanets. In Proceedings of the 31st Annual ACM Symposium on Applied Computing, SAC '16, pages 709-715, New York, NY, USA. ACM.

Lima, D. S., Paula, M. R. P., Roberto, F. M., Cardoso, A. R., and Celestino, J. (2015). Probt: A temporal probabilistic protocol to mitigate the broadcast storm problem in vanets. In 2015 International Conference on Information Networking (ICOIN), pages 7-12.

Panichpapiboon, S. and Pattara-atikom, W. (2012). A review of information dissemination protocols for vehicular ad hoc networks. IEEE Communications Surveys Tutorials, 14(3):784-798.

Paula, M. R. P., Lima, D. S., Roberto, F. M., Cardoso, A. R., and Celestino Jr, J. (2014). A technique to mitigate the broadcast storm problem in vanets. ICN 2014, page 253.

Rezende, C., Boukerche, A., Pazzi, R. W., Rocha, B. P., and Loureiro, A. A. (2011). The impact of mobility on mobile ad hoc networks through the perspective of complex networks. Journal of Parallel and Distributed Computing, 71(9):1189 - 1200. Special Issue on Advancement of Research in Wireless Access and Mobile Systems.

Saeed, T., Mylonas, Y., Pitsillides, A., Papadopoulou, V., and Lestas, M. (2019). Modeling Probabilistic Flooding in VANETs for Optimal Rebroadcast Probabilities. IEEE Transactions on Intelligent Transportation Systems, 20(2):556-570.

Sharshembiev, K., Yoo, S.-M., and Elmahdi, E. (2018). Broadcast storm mitigation from unintentional misbehavior in vehicular ad hoc networks. In 2018 IEEE 8th Annual Computing and Communication Workshop and Conference (CCWC), pages 925-930. IEEE.

Wisitpongphan, N., Tonguz, O. K., Parikh, J. S., Mudalige, P., Bai, F., and Sadekar, V. (2007). Broadcast storm mitigation techniques in vehicular ad hoc networks. IEEE Wireless Communications, 14(6):84-94. 